\title{
Low Power Planar Antenna Inductive Discharge Ion Source
}

\author{
Eric D. Gillman ${ }^{1}$, Paul G. Cummings ${ }^{2}$, and John E. Foster ${ }^{3}$ \\ University of Michigan, Ann Arbor, MI, 48109
}

\begin{abstract}
A $10 \mathrm{~cm}$ stand-alone gridded ion thruster discharge chamber based on a laboratory demonstration model that utilized a planar inductive antenna was designed and fabricated. The source features multipole confinement which has been shown to dramatically improve performance based on significant increases in ion density observed in demonstration tests. The thruster design considerations and the performance goals are discussed. Preliminary thruster discharge operation will be commented upon as well as the future development work necessary to complete the performance assessment.
\end{abstract}

\section{Nomenclature}

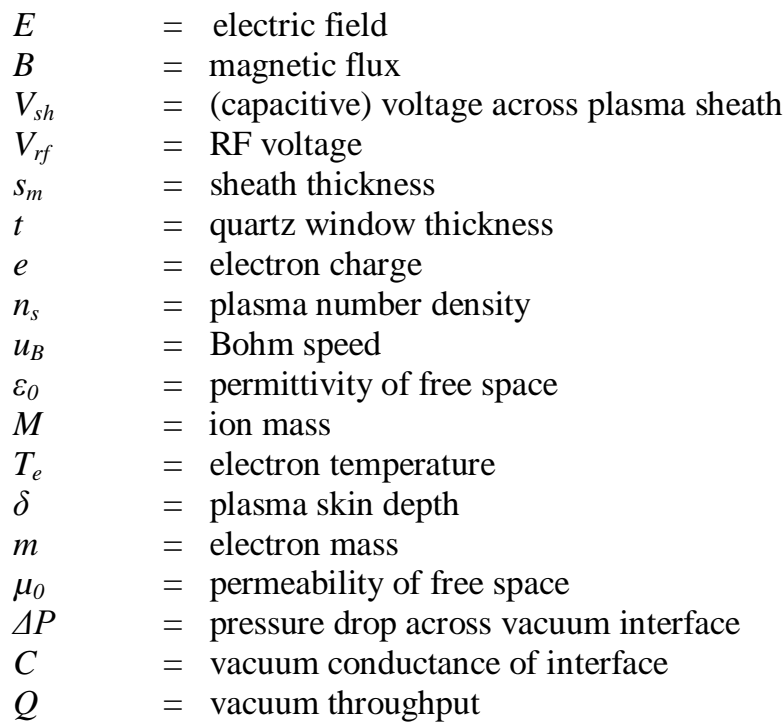

\section{Introduction}

$\mathrm{H}$ igh specific impulse electric propulsion systems enable larger payload fractions for spacecraft launched into orbit. ${ }^{1}$ This technology is literally mission enabling for a variety of missions such as missions to the outer solar system or station keeping applications which result in satellite lifetime extension derived from better thruster utilization of onboard propellant. Electric propulsion systems have seen great success in space missions such as Deep Space 1, SMART-1, and in the ongoing DAWN mission through the use of DC gridded ion thrusters and Hall thrusters. ${ }^{2-5}$ However, these systems are limited in operation time by the cathode lifetime, which is limited by erosion and insert impregnate depletion. The cathode limits the lifetime of DC ion thruster systems to of order 30,000 hours using conventional technologies. ${ }^{3}$ The use of carbon materials in cathode construction may be able to extend lifetime beyond this. Lifetimes significantly greater than 30,000 hours are necessary for space missions such as JIMO, Jupiter Grand Tour, and Pluto orbiter. ${ }^{6-8}$

A great deal of research has been performed in exploring other plasma production approaches that are comparable in discharge efficiency to electron bombardment-based systems, but capable of extended operation in excess of the state of the art. Electric cyclotron resonance (ECR) has been one method of propellant ionization that

${ }^{1}$ Graduate Student, Dept. of Nuclear Engineering, 2355 Bonisteel Blvd., AIAA Member.

${ }^{2}$ Graduate Student, Dept. of Nuclear Engineering, 2355 Bonisteel Blvd., AIAA Member.

${ }^{3}$ Associate Professor, Dept. of Nuclear Engineering, 2355 Bonisteel Blvd., Senior AIAA Member.

American Institute of Aeronautics and Astronautics 092407 
has had recent success. This approach continues to be researched as a possible alternative to DC thrusters. The HAYABUSA mission that was launched in May of 2003 utilized ECR thrusters and has since completed the mission goals of landing on and lifting off of the surface of the asteroid Itokawa before heading back to Earth. The cluster of four ECR ion engines, ' $\mu 10$ 's', have already accumulated over 25,000 hours of operation. The return of the spacecraft to Earth in 2010 will mark the first round trip flight to an asteroid. ${ }^{9}$ The method of ECR ionization utilizes microwave power sources, wire antennas, and waveguides to launch microwaves into the chamber to ionize the discharge plasma as well as provide electrons for ion beam neutralization. In principle, the lifetime of a purely waveguide-configured ECR propulsion system is limited only by the life of the grids and that of the microwave power supply itself. Space qualified microwave power supplies have demonstrated lifetimes over 140,000 hours. ${ }^{10}$

Another electrodeless electric propulsion method that is being considered is the use of radio-frequency (RF) power to generate the discharge plasma. Although these RF ion thrusters typically utilize a cathode-based neutralizer for beam neutralization like that of the DC ion thrusters, this cathode, if configured correctly, will not be exposed to dense plasma or energetic ions. Emission current required is also typically a fraction of that required from a discharge cathode. Therefore it will not undergo the same type of bombardment as the discharge cathode in DC ion thrusters. The RIT series of RF powered plasma thrusters, developed in Germany, have seen recent success. The RIT 10 engine was the first RF thruster successfully tested in space on the European Retrievable Carrier (EURECA), launched in 1992. ${ }^{1}$ A similar thruster, the Radiofrequency Ion Thruster Assembly (RITA), saved the ARTEMIS satellite after it was placed in the wrong orbit by the mission launch vehicle. ${ }^{11} \mathrm{RF}$ driven thrusters have a distinct advantage over microwave systems in that RF power supply efficiency is considerably higher than microwave systems such as TWTs and klystrons, typically approaching that of DC power supplies. Such high power supply electrical efficiency makes RF driven thrusters particularly attractive for low power missions requiring long thrusting times.

The proposed RF system discussed here, as well as the RF driven ion thrusters referred to previously, utilize electromagnetic induction to breakdown and sustain the plasma. High plasma densities can be produced in these systems, typically $10^{12} \# / \mathrm{cm}^{3} .^{3,12-14}$ This inductive mode is achieved in the RIT series of thrusters by wrapping a coil around the outside of a dielectric tube, creating an inductive 'ring' discharge. This RF-driven coil deposits energy in the discharge chamber up to the skin depth. This skin depth is on the order of centimeters and, along with thruster geometry, determines the volume of power deposition. In contrast, the RF-excited plasma source proposed here utilizes a spiral 'stovetop'-like antenna geometry in which the coil lies in a plane above the plasma discharge. This configuration has been used in plasma processing applications to generate high-density, large area, uniform plasmas for substrate processing. This high density, uniform discharge is ideal for ion propulsion. The typical planar spiraled coil sits on top of a dielectric medium that then couples the RF power into the plasma below it, again penetrating a few skin depths. ${ }^{13-14}$ This geometry readily accommodates permanent magnet multipole containment schemes. Such containment tends to reduce the electric field magnitude necessary for gas breakdown and plasma formation, improve confinement of energetic electrons, improve plasma uniformity, and increase plasma density due to better utilization of confined, hot electrons. ${ }^{14}$

These attributes of the inductively coupled plasma in this geometry motivated the investigation and plasma characterization of a magnetically enhanced discharge tested in a Gaseous Electronics Conference (GEC) cell. These tests showed promising results that were particularly advantageous for electric propulsion applications. ${ }^{12}$ High densities $\left(>10^{11} \mathrm{\#} / \mathrm{cm}^{3}\right)$ and high ion currents $(\sim 1 \mathrm{~A})$ were observed at low pressures during the operation of the source on argon. ${ }^{12}$ The present effort focuses on transforming the inductive source as configured in the GEC cell from a plasma processing reactor geometry to a stand-alone laboratory thruster geometry. Design, fabrication, and implementation methodology and issues are discussed herein. In addition, performance targets for the operation of this thruster model will be commented upon as well as the test facilities that will be used for future thruster characterization. The current state of thruster operation and the future steps to be taken in the development of this technology will also be discussed.

\section{Thruster Design}

The chief goal of this design effort is to take the laboratory configuration that was integrated in the GEC test cell and infuse it into a stand-alone system. The thruster design for this geometry consisted of three main parts: the discharge chamber itself, the RF antenna, and the multipole magnetic circuit. For these tests, the discharge chamber is terminated with a grid for assessing ion flow. The modifications necessary in transferring the GEC chamber geometry to a thruster geometry were relatively minimal for the antenna and magnetic circuit. However, the thruster discharge chamber design was by necessity an original design since the chamber had to accommodate mechanical 
requirements such as antenna mounting and power as well as independent gas feed. The fabricated laboratory thruster model is shown in Fig. 1. Here the mechanical design is shown on the left with the hardware realization on the right.

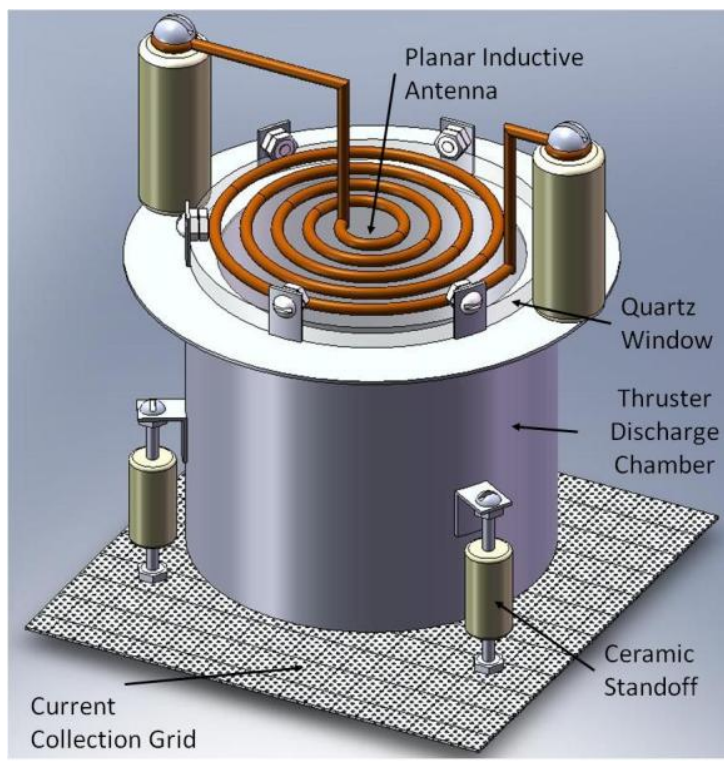

a)

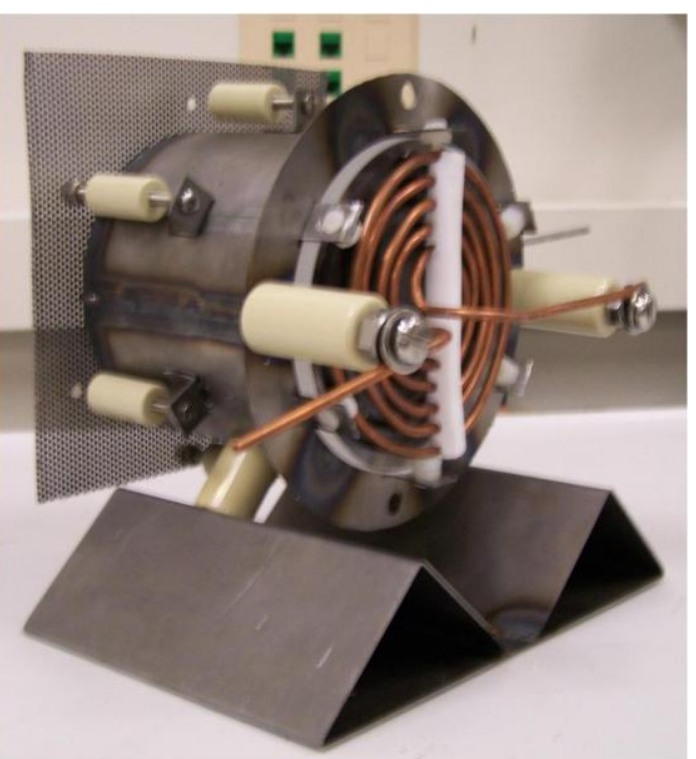

b)

Figure 1. Part (a) shows a design drawing of the thruster including the antenna, quartz window, discharge chamber, and current collection grid. Part (b) shows the fabricated thruster mounted horizontally on a stand for subsequent testing.

\section{A. Thruster Chamber}

The thruster discharge chamber was made from mild steel that was formed into a simple cylindrical shape with modifications at the top of the discharge chamber to hold a $10 \mathrm{~cm}$ diameter, $6.35 \mathrm{~mm}$ thick cylindrical quartz window. The quartz plate serves as a coupling dielectric medium that allows RF energy to enter the discharge chamber while at the same time provides an upstream termination seal and electrical isolation of the antenna from the discharge plasma. The discharge chamber's exit plane was terminated using a steel mesh with about $16 \%(+/-$ $2 \%)$ transparency for ion collection. The grid was mounted using electrically isolated posts mounted to tabs welded on the side of the discharge chamber. The current collection grid can be biased to collect ion current exiting the chamber. The ion exit plane of the thruster is approximately $10 \mathrm{~cm}$ in diameter (comparable to the RIT 10). The height of the discharge chamber is approximately $9 \mathrm{~cm}$, which is somewhat longer than that used in the GEC cell. The gas ring was mounted within the downstream flange of the discharge chamber to allow for reverse gas feed. Reverse gas feed has been shown to have a discharge performance advantage over upstream forward feed configurations. ${ }^{15}$ Under free molecular flow conditions, the residence time within the discharge chamber of a reverse fed gas molecule can be considerably longer than for a similarly launched molecule injected using a forward feed configuration.

The discharge chamber is presently $9 \mathrm{~cm}$ long but it can be reduced with the planar antenna approach. The limitation is essentially the skin depth thickness associated with RF field penetration. Indeed it is possible to reduce chamber length down to about two skin depths $(<4 \mathrm{~cm})$, considerably smaller than DC ion thrusters of similar crosssectional size. However, there is a practical limit to this reduction in length. Reduction in discharge chamber length results in higher neutral loss rates ${ }^{15}$ If the neutral density in the discharge chamber decreases, then it is less likely that an electron will undergo an ionizing collision with a neutral particle before either the electron is lost to the discharge chamber walls or the neutral escapes the chamber. Electrons collected at the wall result in discharge efficiency losses. The same is true of the discharge chamber diameter. That is, when the diameter of the discharge chamber is reduced, electron containment is reduced and therefore efficiency decreases ${ }^{15}$ Maintaining good electron containment and propellant utilization are two of the most challenging issues to be addressed in the scaling of electrical thrusters to low power. While the aforementioned considerations are true for both RF and DC discharges, $\mathrm{RF}$ discharges have a distinct advantage when it comes to size reduction. DC discharges with magnetic confinement rely on maximizing the primary electron containment length. In RF discharges, there is no initial primary electron 
beam injected into the source volume. Rather, an alternating electric field coupled with collisions heats the electrons. Diffusion losses from this heating zone ( skin depth dimensions) can be reduced by the use of an appropriate magnetic field topology. This way electrons can be heated locally to energies necessary to ionize the gas locally, without the need to extend its residence time in the volume to assure an ionizing collision. Additionally, these electrons can accumulate energy via infrequent collisions under low pressure conditions, even at relatively low electric field strengths. In this regard, ionization in the $\mathrm{RF}$ source is somewhat local while in a DC source, ionization is distributed via peripheral ionization processes at the magnetic cusp as well as impact ionization in the volume.

\section{B. Antenna}

The goal of the antenna design was simply to replicate the geometry used in the GEC cell. Therefore, the antenna was designed to mimic the antenna of the GEC cell. Both antennas were made of hollow, $3 \mathrm{~mm}$ outside diameter copper tubing. The center-fed spiral antenna had four full turns. The overall outside diameter of the antenna was

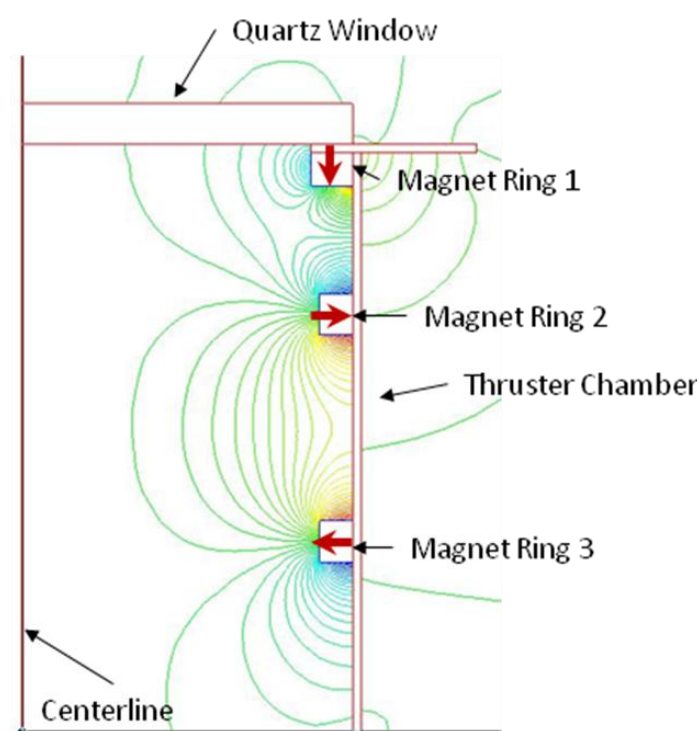

Figure 2. Half plane view of flux lines of the magnetic confinement scheme. Magnet polarity is indicated by the arrow on the magnet rings.

slightly less than $10 \mathrm{~cm}$. The copper tubing was formed into a circle with a steadily changing diameter by hand. A spacer made out of Teflon was later added to hold the spirals of the antenna apart so that adjacent turns could not touch each other to create an electrical short. The antenna was not water cooled. Later experiments will assess antenna temperature as a function of operating time.

\section{Magnetic Circuit Confinement}

The magnetic circuit utilized in the GEC cell experiment needed to be modified somewhat to be integrated into the cylindrical thruster discharge chamber that was designed. The multipole confinement scheme still consisted of 3 rings of permanent samarium-cobalt magnets, but the relative spacing between the rings was modified slightly. In addition, the top magnet ring was located inside the thruster discharge chamber immediately below the quartz window rather than on a flange positioned on the outside, as it was configured in the GEC cell. This ring of magnets was oriented with the north pole vertically downward. The ring cusp magnetic circuit featured alternating polarity magnet rings. The resulting magnetic field contour map is shown in Fig. 2, with the axial profile shown in Fig. 3. It is interesting to note that most of the plasma production will be localized near the quartz window, corresponding to the left side of the axial profile plot. The large magnetic field peak the between the quartz window and exit plane will act to confine the electrons to within about two skin depths of the quartz window and antenna. The confinement of electrons in this area may significantly enhance plasma production. This experimental profile contrasts with typical hollow cathode or filament based multipole sources. In such cases, a monotonically decreasing axial magnetic field gradient at the cathode is desired. This is conducive to primary electron transport to the walls where they encounter the strong magnetic fields associated with the cusps. Here ionization takes place near the walls, predominantly at and between cusps. This peripheral ionization is key to these sources' high ionization efficiency. ${ }^{16}$ The inductive source by contrast relies on heating in the skin depth. A magnetic barrier to confine electrons to the heating zone may therefore be the desired configuration in this case. Testing will include the investigation of modifications to this gradient region in the vicinity of the heating zone to determine the sensitivity of density and uniformity to such changes with the end goal being to either replicate or exceed the performance obtained in the magnetically enhanced GEC cell. 


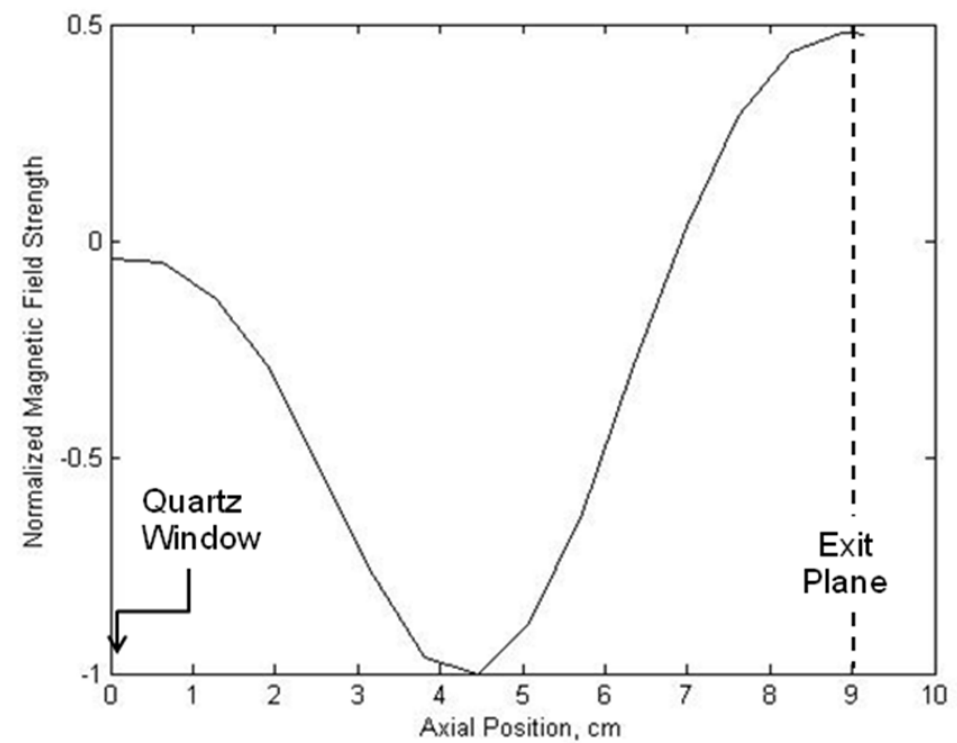

Figure 3. The normalized axial magnetic profile of the thruster discharge chamber. The location of the quartz window and exit plane are indicated.

\section{Performance Targets and Goals}

The aim of the performance of this laboratory model thruster will be to replicate the performance characteristics as reported during testing in the GEC cell. ${ }^{12}$ Once this is achieved, the source will be ready for beam extraction tests. The minimum target performance goals of the thruster are comparable to that of the NASA $8 \mathrm{~cm}$ DC thruster and the RIT $10 \mathrm{~cm}$ thruster. Table 1 lists the performance goals of the NASA 8-cm thruster. Exceeding these goals makes this technology attractive in that it would be applicable to a whole class of science missions requiring ion propulsion. Missions of particular interest are those requiring very long lifetimes ( $\geq 30,000 \mathrm{hrs}$ ). The lifetime limiter in this case would likely be the neutralizer if carbon grids are used to terminate the RF discharge. Based on GEC testing, the discharge efficiency goals should be achievable. It should be pointed out that the GEC source was also capable of producing up to $1 \mathrm{~A}$ of ion current at modest discharge powers on argon. Such high current operation can be utilized for low mass, medium power $(500 \mathrm{~W}<$ Power $<\sim 1 \mathrm{~kW})$ missions. Such higher power processing is possible with the source since the inductive source is not constrained by small cathode thermal and lifetime limits which restrict the useful operating range of these devices. Additionally, a significant performance boost in inductive source performance over that demonstrated in GEC tests is expected when propellant is changed from argon to xenon.

Table 1. Operating parameters for the NASA $8 \mathrm{~cm}$ thruster and GEC RF inductive source performance.

\begin{tabular}{|l|c|c|}
\hline Parameter & $\begin{array}{c}\mathbf{8} \text { cm NASA } \\
\text { Thruster }^{15}\end{array}$ & $\begin{array}{c}\text { GEC Magnetically Enhanced } \\
\text { Inductive Source }^{12}\end{array}$ \\
\hline Lifetime Estimate (hrs.) & $\mathbf{8 0 0 0}$ & $>30,000$ \\
\hline Power Range (kW) & $\mathbf{0 . 1}-\mathbf{0 . 3}$ & $\mathbf{0 . 3 - 1 . 0}$ \\
\hline Discharge Efficiency (W/A) & $\mathbf{2 6 0 - 3 5 0}$ & $\mathbf{2 5 0 - 4 0 0}$ \\
\hline Current (A) & $\mathbf{0 . 1 - 0 . 2}$ & $\mathbf{0 . 2 - 1 . 0}$ \\
\hline
\end{tabular}

Discharge losses measure the amount of power input into the discharge per unit of ion current produced. The thrust produced is proportional to the beam current. Discharge losses in a thruster should be as low as possible to minimize the power supply mass penalty. The primary regime of pressure operation for this thruster will be in the $10^{-4}$ Torr range. The performance target is to achieve discharge losses below $250 \mathrm{~W} / \mathrm{A}$ with the laboratory model at these pressures. GEC tests suggested such a goal is attainable as $250 \mathrm{~W} / \mathrm{A}$ was achieved during those tests. ${ }^{12}$ Furthermore, ion current as high as $1 \mathrm{~A}$ at the exit plane of the thruster at a discharge power of 250 watts was produced with the inductive source investigation, and should also be observable in the stand-alone laboratory model. If these performance goals can be achieved, then this thruster model will offer similar performance and efficiencies

American Institute of Aeronautics and Astronautics 092407 
as small scale DC thrusters, but with extended lifetimes and a broader operating envelope, making medium power missions $(500 \mathrm{~W}-1 \mathrm{~kW})$ within reach as well.

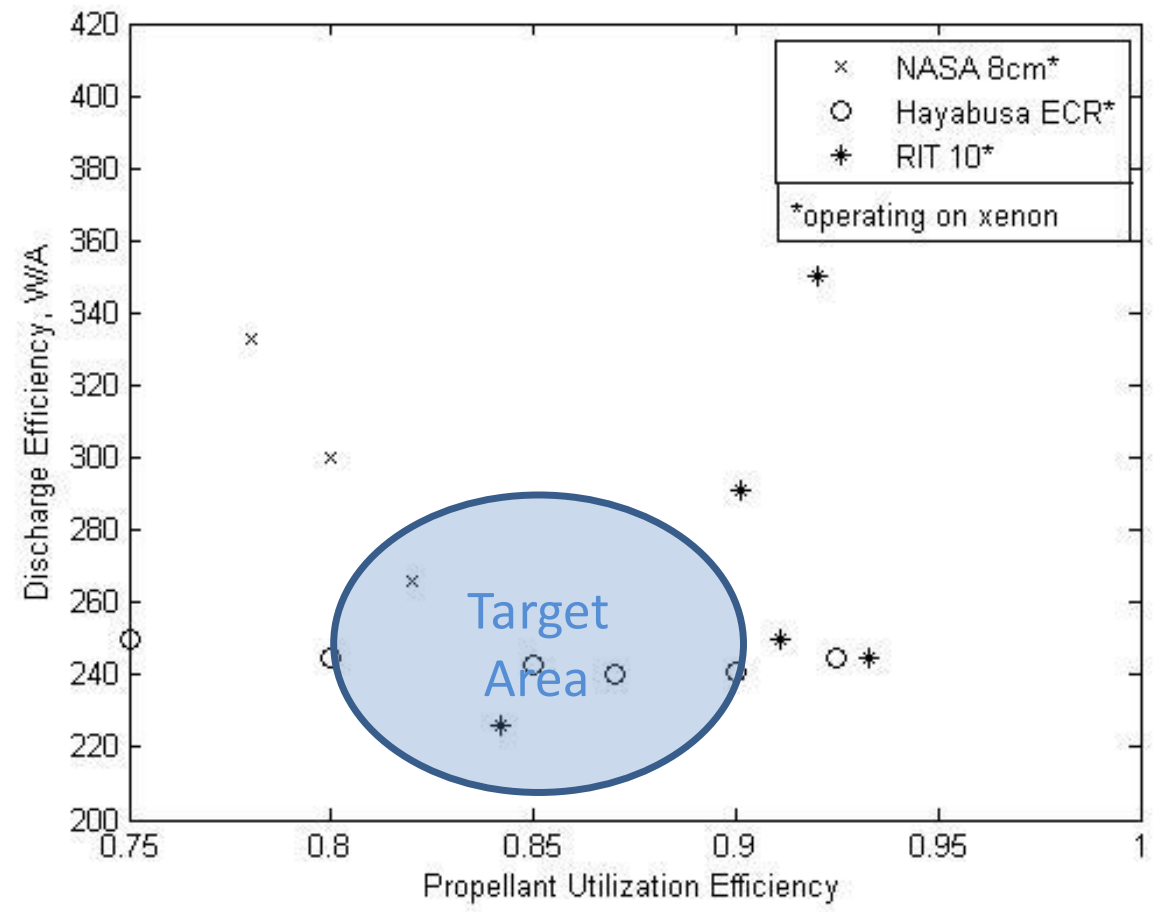

Figure 4. Discharge efficiency as a function of propellant utilization for the NASA $8 \mathrm{~cm}$, Hayabusa ECR, and RIT 10 thrusters operating on xenon as well as the stand-alone laboratory model thruster target area operating on argon.

Figure 4 shows several performance data points as published in literature for several different small-scale electric propulsion thrusters. ${ }^{15,17-21}$ The NASA $8 \mathrm{~cm}$ thruster target operation on xenon is shown, as well as actual operating data for the Hayabusa ' $\mu 10$ ' and RIT 10 thrusters also utilizing xenon as the propellant. As mentioned previously, investigation of the GEC source showed performance down to $250 \mathrm{~W} / \mathrm{A}$, with $1 \mathrm{~A}$ of extracted current. ${ }^{12}$ Based on $^{2}$ this previous data, we would like to be able to operate our laboratory model thruster on argon in the target area highlighted in the figure. The performance objective as illustrated in the highlighted area is to exceed the performance of the NASA $8 \mathrm{~cm}$ thruster and RIT 10 thruster, and at the same time provide much greater lifetimes than small-scale DC thrusters. In addition to the goal of providing a high-efficiency and extended lifetime alternative to small-scale DC thrusters, this thruster is expected to have a wider power processing envelope, encompassing both low and medium power ranges.

The RF inductive discharge laboratory model thruster effort will carefully assess utilization, discharge efficiency and discharge uniformity by means of discharge only operation. The flow rates will be corrected using the approach of Brophy. ${ }^{22}$ Continuing work will involve actual beam extraction. Beam extraction with this source has the advantage that the RF supply may not be required to float at high voltage. This peculiar attribute is associated with antenna isolation via the quartz plate. The working gas in this analysis will initially be argon, which has a higher ionization potential than xenon. Performance is expected to improve significantly with subsequent testing on xenon gas. Significant performance improvements were measured with the RIT 10 operating on xenon compared to argon. ${ }^{23}$

\section{Experimental Setup}

\section{A. Theory and Background}

The production of plasma via inductive discharge is physically similar to the coupling mechanism that takes place inside a transformer. However in an inductive discharge the "secondary coil" is the plasma. A time-varying current in the antenna produces a time-varying magnetic field, which produces an electric field according to Faraday's law: 


$$
\nabla \times \vec{E}=\frac{d \vec{B}}{d t}(\text { Ref. 24) }
$$

The magnetic field and induced electric field for a planar spiral "pancake" antenna are illustrated below in Fig. 5.

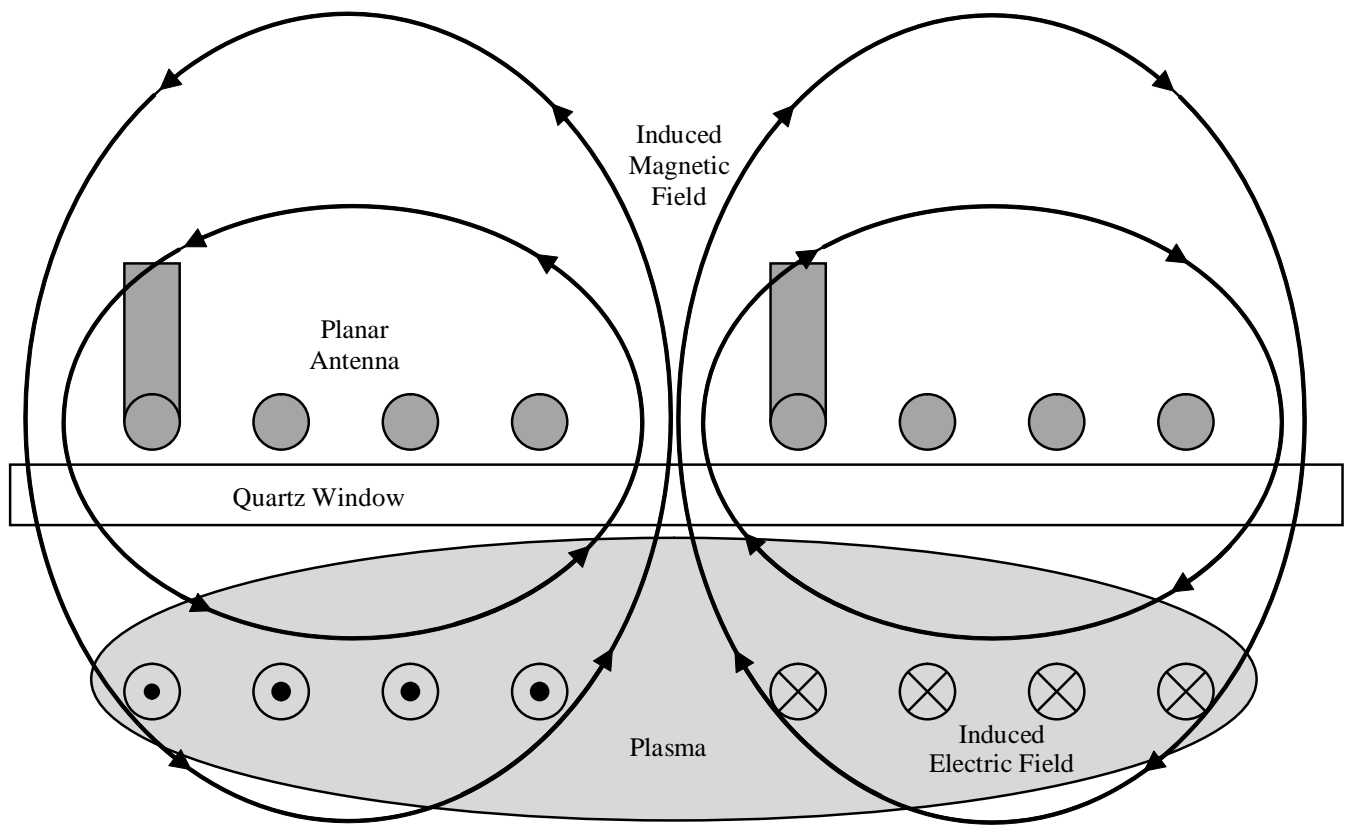

Figure 5. Inductive coupling of the planar antenna with the plasma through the quartz window.

The depth of penetration of the resulting electromagnetic wave at low pressures is given by:

$$
\delta=\sqrt{\frac{m}{e^{2} \mu_{0} n_{s}}}(\text { Ref. 25) }
$$

where $m$ is the electron mass, $e$ is the electron charge, $\mu_{0}$ is the permeability of free space, and $n_{s}$ is the plasma density. Note that here, "low pressure" means that the RF frequency exceeds the electron-neutral collision frequency. The RF antenna will be excited at a frequency of $13.56 \mathrm{MHz}$, which is significantly larger than the electron-neutral collision frequency at the targeted discharge chamber operating pressure range of $\sim 10^{-3}-10^{-4}$ Torr. For plasma densities of $10^{11} \mathrm{\#} / \mathrm{cm}^{3}$, Eq. (2) yields a skin depth of $\sim 1.7 \mathrm{~cm}$. Since the lower limit on chamber depth is set by the skin depth, very shallow, low-mass configurations should be possible.

\section{Capacitive vs. Inductive Coupling}

In addition to the inductively coupled mode presented above, the antenna, which is at high voltage, can also couple to the plasma capacitively. The degree to which capacitive coupling occurs varies inversely with plasma density; it is less significant at higher plasma densities. Since the density of the plasma is dependent on the RF power, the effects of capacitive coupling decrease as the RF power is increased. To determine the degree of capacitive coupling, note that the sheath capacitance per unit area scales as $\varepsilon_{0} / s_{m}$, where $s_{m}$ is the sheath thickness and $\varepsilon_{0}$ is the permittivity of free space. The capacitance of the quartz window scales as $\varepsilon_{0} / t$, where $t$ is the thickness of the quartz window. Using the capacitive voltage divider formula, the potential drop across the sheath is:

$$
\tilde{V}_{s h}=V_{r f} \frac{s_{m}}{t+s_{m}}(\text { Ref. 25) }
$$

where $V_{s h}$ is the potential drop across the sheath and $V_{r f}$ is the RF voltage. The sheath thickness can be determined using the well-known Child-Langmuir law:

$$
\bar{J}_{i}=e n_{s} u_{B}=0.82 \varepsilon_{0}\left(\frac{2 e}{M}\right)^{\frac{1}{2}} \frac{\tilde{V}_{s h^{2}}}{s_{m}{ }^{2}}(\operatorname{Ref} .25)
$$




$$
e n_{s} u_{B}=0.82 \varepsilon_{0}\left(\frac{2 e}{M}\right)^{\frac{1}{2}} \frac{1}{s_{m}{ }^{2}}\left(V_{r f} \frac{s_{m}}{t+s_{m}}\right)^{\frac{3}{2}}(\text { Ref. 25) }
$$

where $u_{B}$ is the Bohm velocity and $M$ is the ion mass. Assuming $t>>s_{m}$, this equation becomes:

$$
s_{m} \approx\left(\frac{0.82 \varepsilon_{0}}{e n_{s} u_{B}}\right)^{2}\left(\frac{2 e}{M}\right)\left(\frac{V_{r f}}{t}\right)^{3}(\text { Ref. 25) }
$$

Assuming typical plasma values $\left(n_{s}=10^{11} \# / \mathrm{cm}^{3}, T_{e}=5 \mathrm{eV}, u_{B}=3.5^{*} 10^{3} \mathrm{~m} / \mathrm{s}\right)$, knowing that the thickness of the quartz window is $6.4 \mathrm{~mm}$, and assuming an RF voltage of 2000 Volts, the sheath thickness is $2.56 \mathrm{~mm}$. This gives a sheath potential of 571 volts, or about $\sim 29 \%$ of the RF voltage. It should be pointed out that while the presence of capacitive fields can give rise to sputtering and overall higher energy losses to the wall, it is necessary for initial thruster startup. In fact, it is believed that the capacitive coupling is responsible for the self starting nature of this type of discharge.

\section{Magnetic Circuit}

The use of static magnetic fields to expand the pressure range at which an RF source can operate is well established. For this thruster design, 3 ring cusps were incorporated into the magnetic circuit of the discharge chamber (see previous section on thruster design for details). Static magnetic fields improve discharge operation by reducing the effective wall surface area. This reduces diffusion losses and increases the residence time of heated electrons, increasing the overall efficiency of the thruster. The magnetic circuit utilized here also functions to confine electrons to the heating zone or skin depth region as well. It is believed that this approach can enhance discharge performance, particularly at low flow rates and correspondingly low internal discharge chamber pressures.

3. Discharge Chamber Pressure

Because feed gas is injected into the thruster, the thruster discharge chamber pressure will be higher than the background pressure. In this work, only vacuum chamber pressure was monitored. The pressure inside the discharge chamber, however, can be estimated. The pressure difference between the discharge chamber and the surrounding volume of the vacuum chamber is given by:

$$
\triangle P C=Q
$$

where $\Delta P$ is the pressure difference between the tank and the discharge chamber, $C$ is the vacuum conductance of the interface between the discharge chamber and the tank, and $Q$ is the throughput of the system. The interface (the extraction grid) can be modeled as a collection of thin apertures (grid holes) in parallel. The number of aperture is given by the area density of holes $\left(\sim 17.4\right.$ holes $\left./ \mathrm{cm}^{2}\right)$ times the grid area $\left(81.1 \mathrm{~cm}^{2}\right)$, which yields 1411 holes in the grid. The conductance of an individual hole is given by:

$$
C=12.1 * \frac{D^{3}}{L} \alpha(\text { Ref. 26) }
$$

where $D$ is the diameter in $\mathrm{cm}, L$ is the depth in $\mathrm{cm}$, and $\alpha$ is the Clausing factor, given by:

$$
\alpha=\frac{15\left(\frac{L}{D}\right)+12\left(\frac{L}{D}\right)^{2}}{20+38\left(\frac{L}{D}\right)+12\left(\frac{L}{D}\right)^{2}}(\text { Ref. 26) }
$$

The grid holes had diameters of $1 \mathrm{~mm}$, and depths of $0.85 \mathrm{~mm}$. This led to a Clausing factor of 0.42 , resulting in a conductance of $59.8 \mathrm{ml} / \mathrm{s}$ per hole. The total conductance of the grid was therefore $84.4 \mathrm{l} / \mathrm{s}$. A typical flow rate of 12 sccm corresponds to about 0.15 Torr-1/s. This resulted in a pressure difference between the discharge chamber and the tank of $1.8 * 10^{-3}$ Torr. Therefore at $12 \mathrm{sccm}$, pressure in the discharge chamber exceeded the pressure in the vacuum tank by a factor of 30 for the discharge-only testing.

\section{B. Vacuum Chamber}

The vacuum chamber used for this experiment was a $64 \mathrm{~cm}$ diameter, $190 \mathrm{~cm}$ long cylindrical stainless steel chamber, shown below in Fig. 6. Initially, the chamber was pumped using a Trivac rotary vane roughing pump with a trap which had a pumping speed of $25.3 \mathrm{l} / \mathrm{s}$. Later experiments made use of a Pfeiffer TPU 510 turbopump with a pumping speed of $500 \mathrm{l} / \mathrm{s}$, which was backed by the roughing pump. Ultimate pressures of $\sim 10^{-5}$ Torr were achievable in this configuration. 


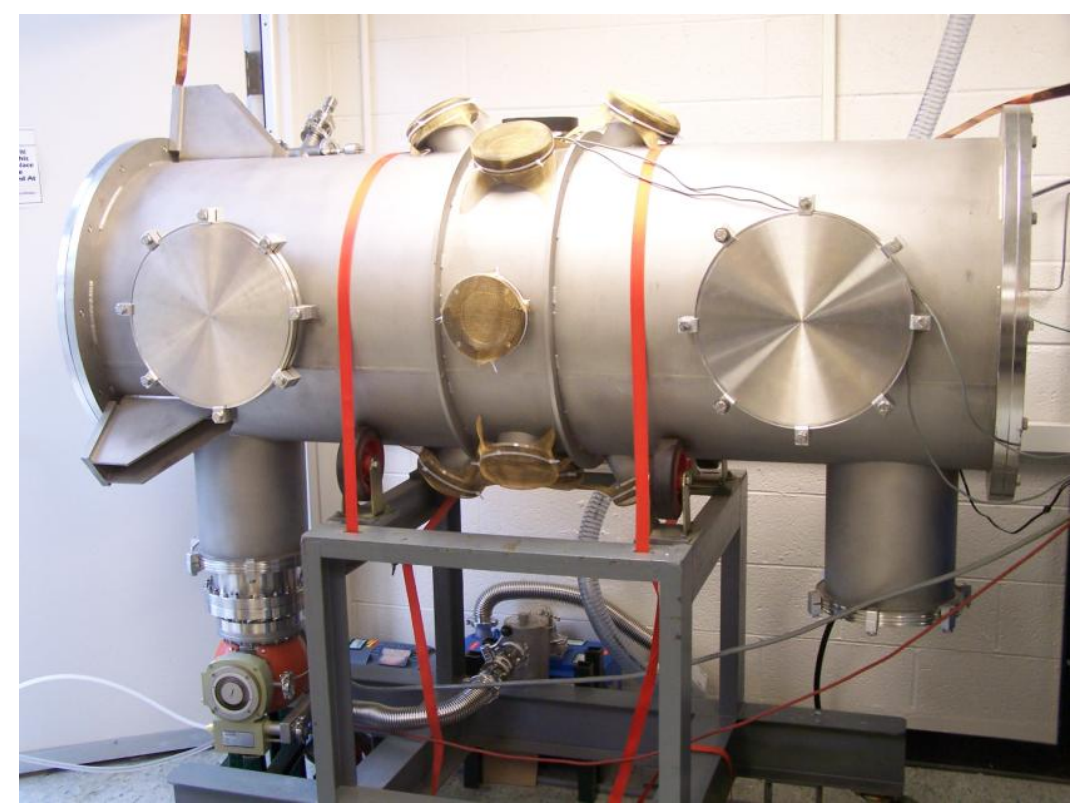

Figure 6. The vacuum chamber used for testing of the $10 \mathrm{~cm}$ laboratory model thruster.

Gas flow into the chamber was achieved via an argon line fed directly into the thruster discharge chamber. Gas flow was controlled via a precision mass flow meter.

\section{Diagnostics and Power}

Pressure measurements were made using a thermocouple gauge at roughing pressures (above $~ 30 \mathrm{mTorr}$ ) and a Pirani gauge at lower pressures (below $\sim 30$ mTorr). During setup optimization, due to RF interference, both of these gauges were turned off when the RF power was activated. Here chamber pressure at a given flow was recorded just prior to discharge initiation.

The thruster was powered by an RF power supply via the circuit shown schematically in Fig. 7. RF power was transmitted to the thruster antenna via a high-voltage vacuum feedthrough. A manually-tunable matching circuit was used to match the circuit impedances and minimize reflected power. A DC bias was placed on the thruster grid to measure the collected ion grid current. A low-pass filter was used to help filter out the RF noise in the DC bias signal; however, even with the low-pass filter present, finite RF noise interfered with the grid bias DC power supply. The RF noise is currently being addressed. For the initial tests, to avoid the power supply interference issue, a battery pack was used to bias the grid at $-24 \mathrm{~V}$.

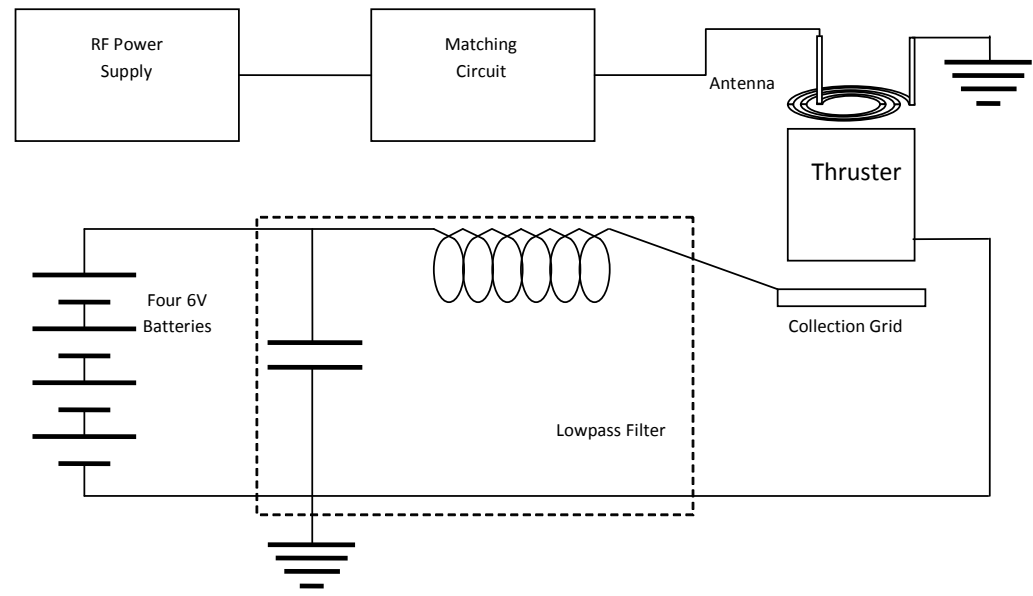

Figure 7. The RF power circuit and ion current collection circuit.

American Institute of Aeronautics and Astronautics 
The matching circuit, used to match the impedance of the system to that of the power supply, is shown schematically in Fig. 8. Tuning was effected by adjusting the values of the two variable capacitors until reflected power was minimized.

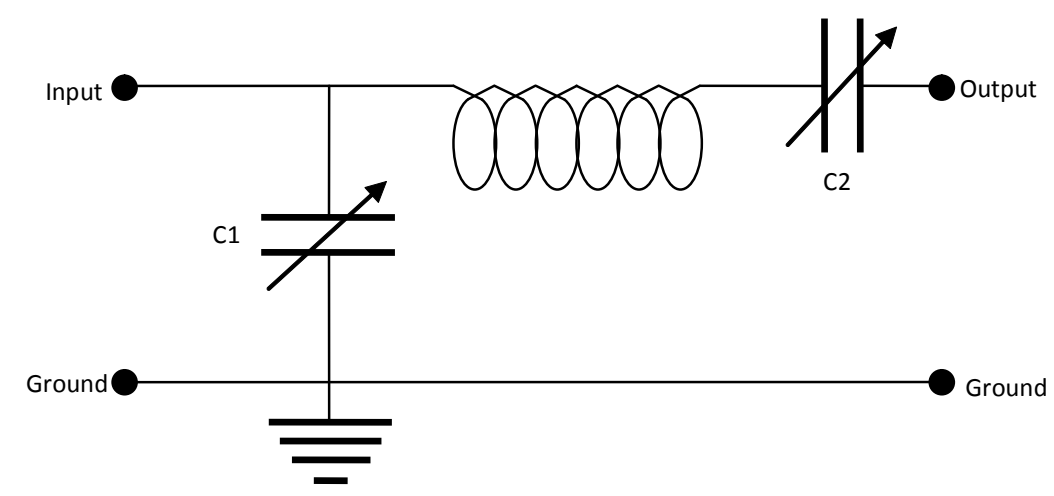

Figure 8. Matching network circuit components used to match the RF power load to the discharge.

\section{Preliminary Check Out and Current Status}

The standalone plasma source has been fabricated and is presently being prepared for testing. During initial checkout tests of the source, in addition to the discharge present in the discharge chamber, parasitic RF discharges were also observed on surfaces associated with the power feed line. Capacitive coupling associated in part with inadequate grounding was identified as the basis of formation for these discharges. These issues, which are currently being mitigated, precluded performance characterization by the time of this writing.

The source was self starting at a chamber pressure of 30 mTorr. Breakdown occurred at less than $20 \mathrm{~W}$ input RF power. Figure 9 illustrates discharge operation. The intense, white glow observed in the thruster chamber indicates that the antenna is inductively coupling into the discharge at RF powers below $250 \mathrm{~W}$. The discharge appears to be fairly dense and uniform. Application of negative bias voltage to the grid gave rise to the formation of a beam as shown in the figure. The beam is made visible through its interaction with the ambient gas.

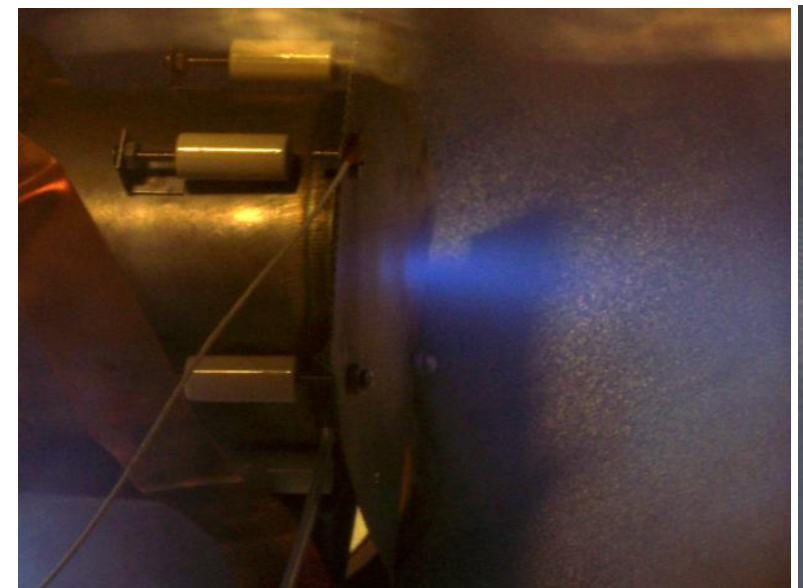

a) photograph of the discharge chamber while operating in the inductively coupled regime.

\section{Conclusion}

A novel RF thruster is being pursued as an alternative to a hollow cathode based discharge which is inherently limited by the cathode lifetime. The source differs from the RIT engines in that it features a planar antenna which allows for compact, high density plasma production. The thruster was designed to duplicate the inductive discharge produced in a GEC cell with the same type of antenna and similar magnetic confinement. The performance projections of this thruster have been outlined based on test results of a previous study. These performance projections are also compared with the NASA $8 \mathrm{~cm}$ performance targets. ${ }^{12,15}$ Discharge efficiencies and utilization 
higher than current DC sources should be attainable based on previous laboratory tests. A key objective of this ongoing work is to replicate such results with the stand-alone thruster. The expected thruster power range is 0.1 to 1 $\mathrm{kW}$, thereby satisfying a number of mission possibilities. To validate these projections, a stand-alone laboratory model thruster utilizing a planar antenna was designed and fabricated. The laboratory stand-alone thruster is operational, demonstrating inductive coupling and self-starting plasma formation. As of this writing, full up performance characterization was delayed to resolve parasitic RF discharge formation (outside thruster) associated with capacitive coupling. Such discharges are power sinks and can give rise to error in performance assessments.

\section{References}

${ }^{1}$ Groh, K. H., and Loeb, H. W., "State of the Art of Radio-Frequency Ion Sources for Space Propulsion," Review of Scientific Instruments, Vol. 65, No. 5, May 1994, pp. 1741-1744.

${ }^{2}$ Rayman, M. D., Varghese, P., Lehman, D. H., and Livesay, L. L., "Results From the Deep Space 1 Technology Validation Mission," 50 th International Astronautical Congress, IAA-99-IAA.11.2.01, Oct. 1999.

${ }^{3}$ Sengupta, A., Brophy, J. R., and Goodfellow, K. D., "Status of the Extended Life Test of the Deep Space 1 Flight Spare Ion Engine After 30,352 Hours of Operation," $39^{\text {th }}$ AIAA/ASMA/SAE/ASEE Joint Propulsion Conference and Exhibit, AIAA-20034558, July 2003.

${ }^{4}$ Koppel, C. R., Marchandise, F., Prioul, M., Estublier, D., and Darnon, F., "The SMART-1 Electric Propulsion Subsystem Around the Moon: In Flight Experience," $41^{s t}$ AIAA/ASME/SAE/ASEE Joint Propulsion Conference \& Exhibit, AIAA-20053671, July 2005.

5Rayman, M. D., "Dear Pseudawnyms," DAWN Journal [online mission journal], URL: http://dawn.jpl.nasa.gov/mission/journal_5_27_08.asp [cited 25 June 2008].

${ }^{6}$ Oleson, S., and Katz, I., "Electric Propulsion for Project Prometheus," 39 ${ }^{\text {th }}$ AIAA/ASME/SAE/ASEE Joint Propulsion Conference \& Exhibit, AIAA-2003-5279, July 2003.

${ }^{7}$ Rawlin, V. K., Soulas, G. C., Williams, G. J., and Roman, R. F., "High Specific Impulse, High Power Ion Engine Operation," $38^{\text {th }}$ AIAA/ASME/SAE/ASEE Joint Propulsion Conference \& Exhibit, AIAA-2002-3838, July 2002.

${ }^{8}$ Patterson, M. J., "Low-Power Ion Thruster Development Status," $34^{\text {th }}$ AIAA/ASME/SAE/ASEE Joint Propulsion Conference \& Exhibit, AIAA-1998-3347, July 1998.

${ }^{9}$ Kuninaka, H., Nishiyama, K., Funaki, I., Yamada, T., Shimizu, Y., and Kawaguchi, J., "Powered Flight of HAYABUSA in Deep Space," 42 $2^{\text {nd }}$ AIAA/ASME/SAE/ASEE Joint Propulsion Conference \& Exhibit, AIAA-2006-4318, July 2006.

${ }^{10}$ Hansen, J. W., "U.S. TWTs from 1 to 100 GHz," Microwave Journal, Vol. 32, Sep. 1989, pp. 179-183.

${ }^{11}$ Notarantonio, A., Killinger, R., and Amorosi, L., "Ion Propulsion System Saves ARTEMIS Satellite," 54 $4^{\text {th }}$ International Astronautical Congress of the International Astronautical Federation, the International Academy of Astronautics, and the International Institute of Space Law, IAC-03-S.4.01, Sep. 2003.

${ }^{12}$ Foster, J. E., Gillman, E. D., Sommers, B., Weatherford, B., "Magnetically Enhanced Inductive Source for Electric Propulsion Applications," 43 ${ }^{\text {rd }}$ AIAA/ASME/SAE/ASEE Joint Propulsion Conference \& Exhibit, AIAA-2007-5294, July 2007.

${ }^{13}$ Hopwood, J., Guarieri, C. R., Whitehair, S. J., and Cuorno, J. J., "Electromagnetic Fields in a Radio-Frequency Induction Plasma," Journal of Vacuum Science and Technology A, Vol. 11, No. 1, Jan./Feb. 1993, pp. 147-151.

${ }^{14}$ Hopwood, J., "Review of Inductively Coupled Plasmas for Plasma Processing," Plasma Sources Science and Technology, Vol. 1, No. 2, April 1992, pp. 109-116.

${ }^{15}$ Patterson, M. J., Grisnik, S. P., and Soulas, G. C., "Scaling of Ion Thrusters to Low Power," $25^{\text {th }}$ International Electric Propulsion Conference, IEPC 97-098, May 1998.

${ }^{16}$ Gauthereau, C., Matthieussent, G., "Plasma Density Profiles in Discharges Surrounded By Magnetic Multipole Walls," Physics Letters, Vol. 102A, No. 5-6, 21 May 1984, pp. 231-234.

${ }^{17}$ Kuninaka, H., Kazutaka, N., Shimizu, Y., and Toki, K., "Flight Status of Cathode-Less Microwave Discharge Ion Engines Onboard HAYABUSA Asteroid Explorer," 40 th AIAA/ASME/SAE/ASEE Joint Propulsion Conference \& Exhibit, AIAA-20043438, July 2004.

${ }^{18}$ Funaki, I., Kuninaka, H., and Toki, K., "Plasma Characterization of a 10-cm Diameter Microwave Discharge Ion Thruster," Journal of Propulsion and Power, Vol. 20, No. 4, July-August 2004.

${ }^{19}$ Walther, R. J., Geisel, J. E., Pinks, W. F., Rothermel, K. H., Trojan, F. M., and Loeb, H. W., "Scaling Laws of RadioFrequency Ion Thrusters," AIAA $11^{\text {th }}$ Electric Propulsion Conference, AIAA-75-367, March 1975.

${ }^{20}$ Groh, K. H., and Loeb, H. W., "State-of-the-Art of Radio-Frequency Ion Thrusters," AIAA/ASME/SAE/ASEE 25 th Joint Propulsion Conference, AIAA-89-2381, July 1989.

${ }^{21}$ Loeb, H. W., "Recent Works on Radio Frequency Ion Thrusters," AIAA $8^{\text {th }}$ Electric Propulsion Conference, AIAA-701102, Aug.-Sept. 1970.

${ }^{22}$ Brophy, J. R., "Simulated Ion Thruster Operation Without Beam Extraction," AIAA/DGLR/JSASS $21^{\text {st }}$ International Electric Propulsion Conference, AIAA-90-2655, July 1990.

${ }^{23}$ Groh, K. H., Blum, O., Rado, H., and Loeb, H. W., "Inert Gas Radio-Frequency Thruster RIT 10," Princeton/AIAA/DGLR $14^{\text {th }}$ International Electric Propulsion Conference, 79-2100, AIAA, New York, N.Y., 1979.

${ }^{24}$ Griffiths, D. J., Introduction to Electrodynamics, $3{ }^{\text {rd }}$ ed., Prentice-Hall, Inc., Upper Saddle River, NJ, 1999, Chaps. 7.

${ }^{25}$ Lieberman, M. A., and Lichtenberg, A. J., Principles of Plasma Discharges and Materials Processing, $2^{\text {nd }}$ ed., John Wiley \& Sons, Inc., Hoboken, NJ, 2005, Chaps. 6, 11, 12. 
${ }^{26}$ Guthrie, A., and Wakerling, R. K., Vacuum Equipment and Techniques, $1^{\text {st }}$ ed., McGraw-Hill Book Company, Inc.., New York, 1949, Chap. 1. 